This is a postprint version of the following published document:

López Boada, M. J., López Boada, B., Díaz, V. (2017). A novel frequency dependent model based on trigonometric functions for a magnetorheological damper. Meccanica, pp. 1-15.

DOI: $10.1007 / \mathrm{s} 11012-017-0632-2$

(c) Springer Science+Business Media Dordrecht 2017 


\title{
A novel frequency dependent model based on trigonometric functions for a magnetorheological damper
}

\author{
Maria Jesus L. Boada - Beatriz L. Boada - Vicente Diaz
}

\begin{abstract}
In this paper, a novel frequency dependent MR damper model based on trigonometric functions is proposed. The model presents the following advan-tages in comparison with other previously proposed models: (1) it is based on algebraic functions instead of differential equations, so that it does not present convergence problems when noisy inputs from exper-imental measurements are used; (2) the number of parameters is reasonable, so that it makes the model computationally efficient in the context of parameter identification and (3) the model has to take into account the variation of the parameters as a function, not only of the applied current but also of the frequency of excitation. Experimental results confirm that the proposed frequency dependent MR damper model improves the accuracy of the model in force simulation.
\end{abstract}

M. J. L. Boada $(\varangle) \cdot$ B. L. Boada · V. Diaz

Mechanical Engineering Department,

Universidad Carlos III de Madrid, Avda. de la

Universidad, 30,

28911 Leganes, Madrid, Spain

e-mail: mjboada@ing.uc3m.es

B. L. Boada

e-mail: bboada@ing.uc3m.es

V. Diaz

e-mail: vdiaz@ing.uc3m.es
Keywords: Magnetorheological damper .

Frequency dependent model . Trigonometric functions - Experimental test - Genetic algorithms - Neural networks

\section{Introduction}

Nowadays, magnetorheological (MR) dampers are receiving significant attention in applications of vibration isolation due to the controllable characteristics of MR fluid [17, 25, 27]. An MR damper is a semi-active device that contains a hydraulic fluid whose viscosity is controllable upon the application of an external magnetization field. MR dampers contain suspensions of micron-size, magnetizable particles in an oil-based fluid. In the presence of magnetic fields, these fluid particles become aligned with the field, dramatically increasing the fluid viscosity as an effective damping. Along with their variable damping force, MR dampers have been shown to have very low response times, fault-safe and low power-consumption $[7,13]$. However, a major drawback is their inherent non-linear and hysteretic dynamics. In this case, it is necessary to develop an appropriate control strategy which is practically implementable when an accurate model for MR dampers is available [21].

There are several MR damper models proposed in the literature, in order to describe their non-linear and hysteretic responses $[3,4,8,14,16,28]$. One of the most extensively used models to describe the non- 


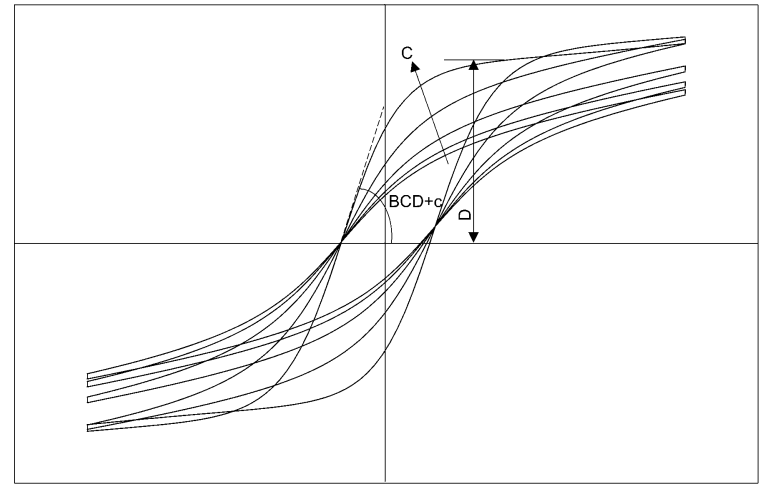

(a) Parameter C

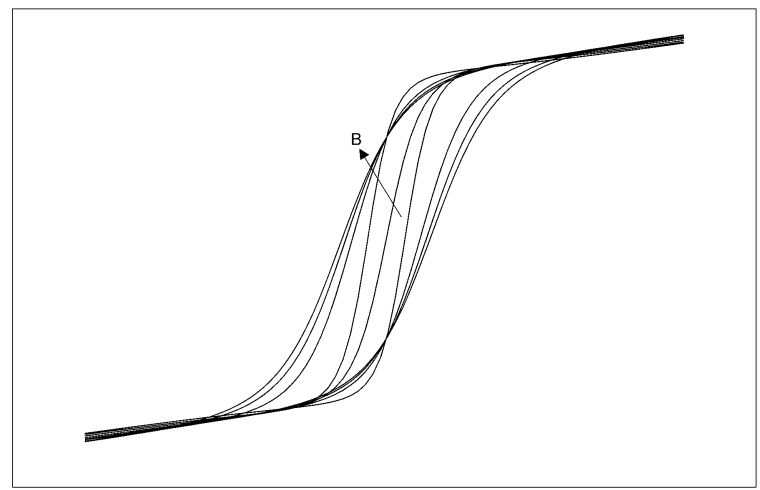

(c) Parameter B

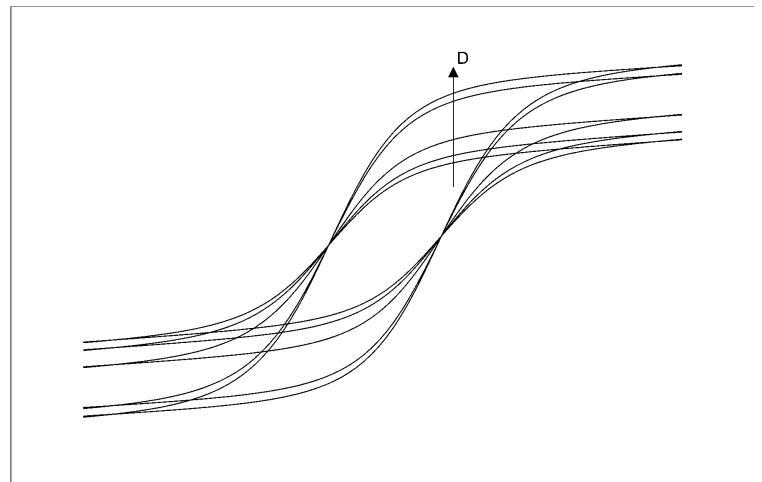

(b) parameter D

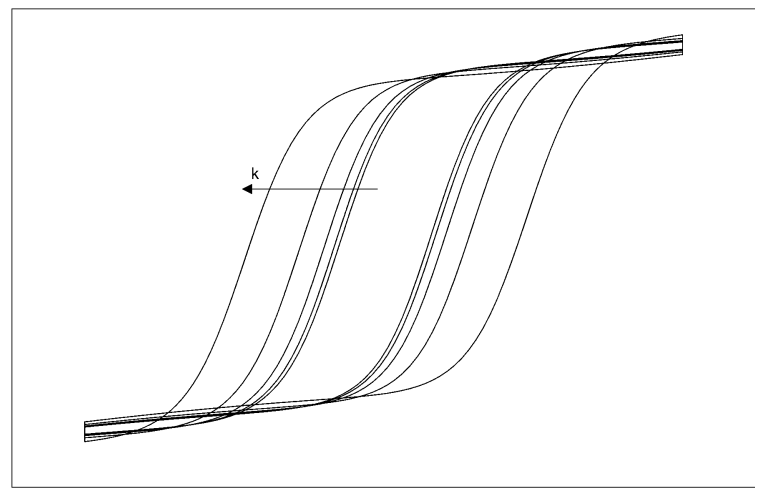

(d) parameter $\mathrm{k}$

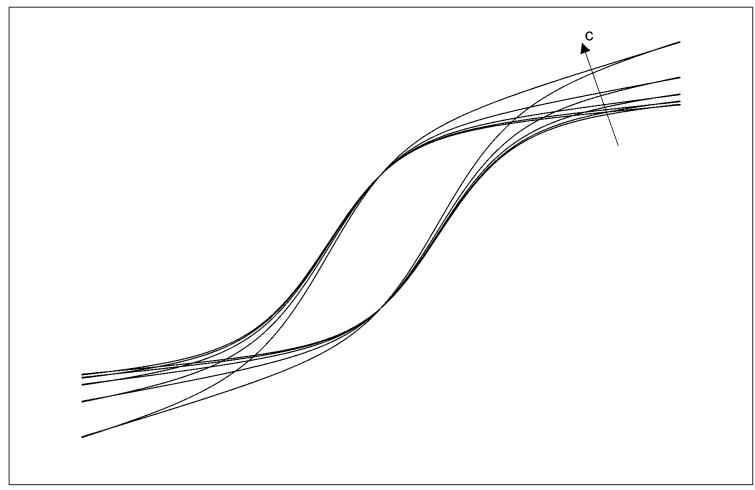

(e) Parameter c

Fig. 1 Influence of the parameters of the model a Parameter C, b Parameter D, $\mathbf{c}$ Parameter B, $\mathbf{d}$ Parameter k, $\mathbf{e}$ Parameter c

linear hysteretic behaviour of a MR damper is the Bouc-Wen model, proposed by Spencer et al. [24]. This model proposes first-order differential equations to desscribe the response of an MR damper over a wide range of loading under a voltage. In this case, the force of the MR damper is expressed as:

$\mathrm{f}=\mathrm{c}_{1} \dot{y}+k_{1}\left(x-x_{0}\right)$

$$
\begin{aligned}
& \dot{y}=\frac{\alpha z+c_{0} \dot{x}+k_{0}(x-y)}{c_{0}+c_{1}} \\
& \dot{z}=-\gamma|\dot{x}-\dot{y}| z|z|^{n-1}-\beta(\dot{x}-\dot{y})|z|^{n}+A(\dot{x}-\dot{y})
\end{aligned}
$$

where $\dot{x}$ is the piston velocity, $x$ is the piston displacement, $\alpha$ is the Bouc-Wen model parameter related to the MR material yield stress, $k_{1}$ is 


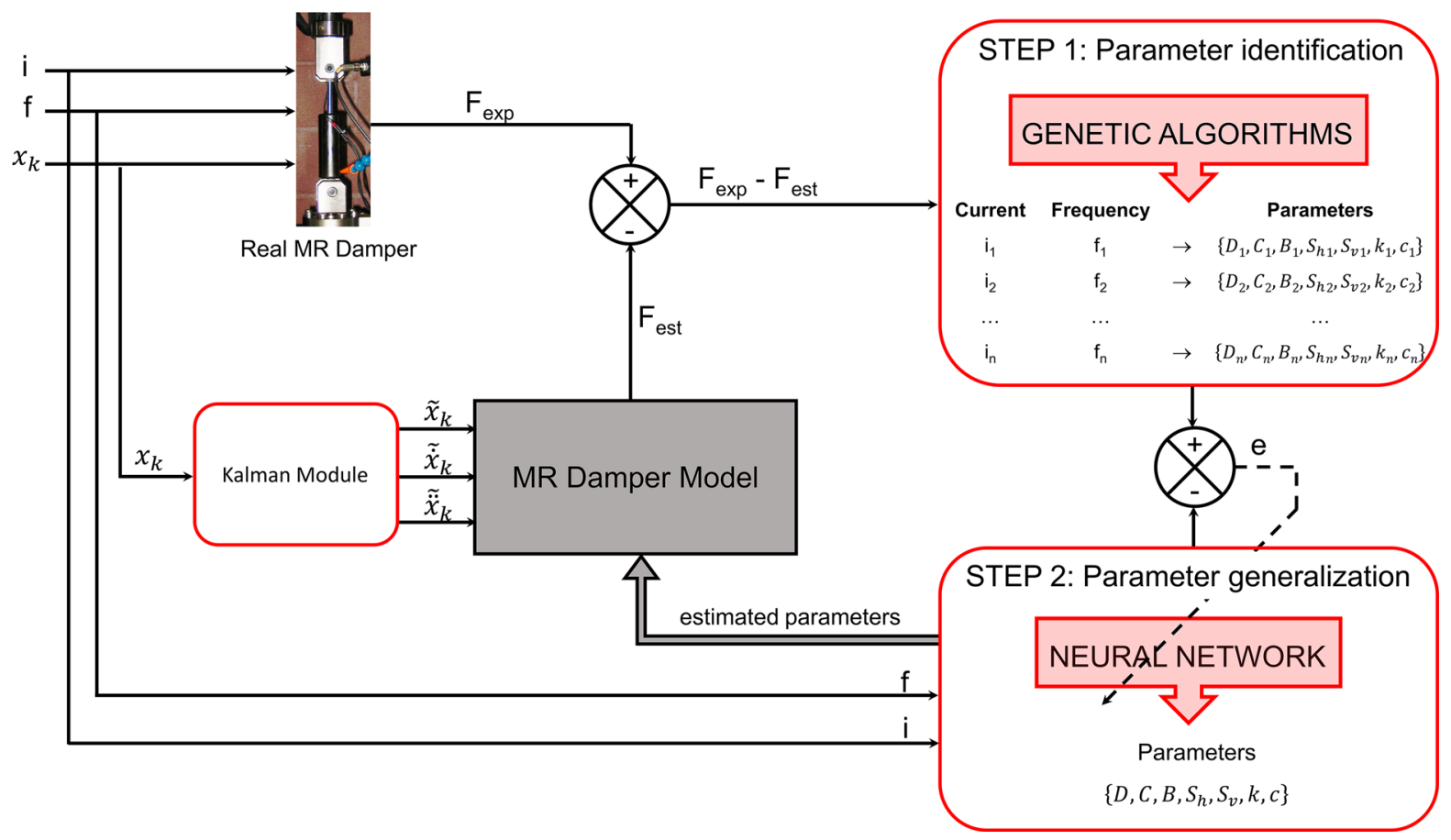

Fig. 2 Scheme of modelling for MR damper

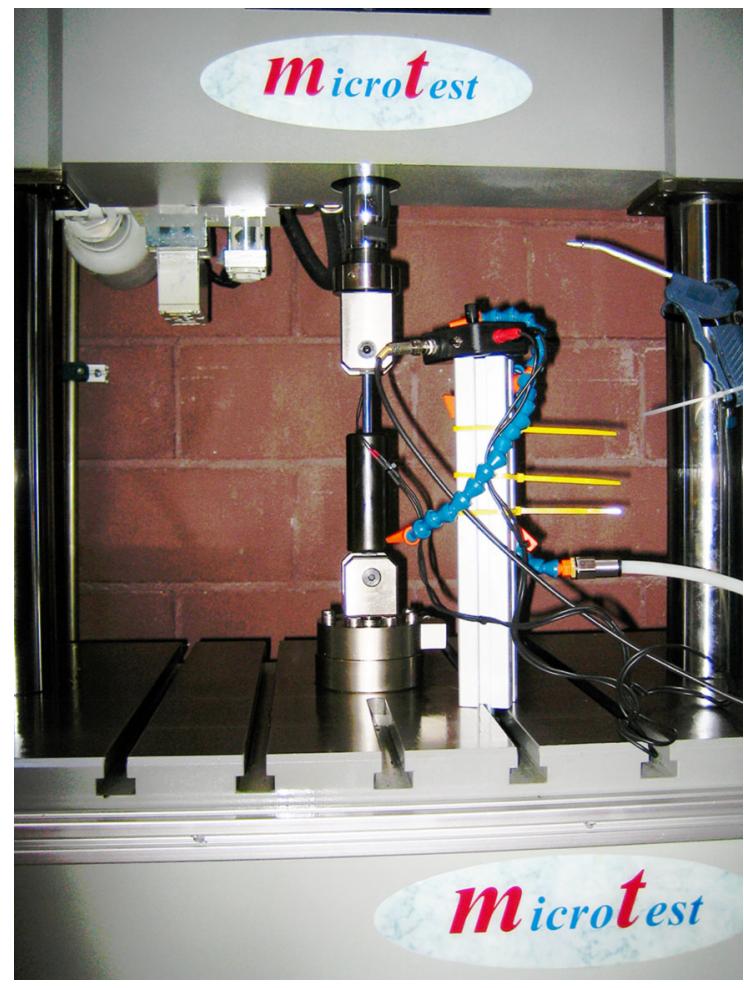

Fig. 3 Damper test machine accumulator stiffness, $c_{1}$ is the dash-pot damping coefficient, $k_{0}$ is present to control the stiffness at large velocities, $c_{0}$ is the viscous damping observed at larger velocities, $z$ is the hysteretic deformation of the model, $y$ is an internal dynamical variable and $A, \beta$ and $\gamma$ are the Bouc-Wen model parameters. Spencer et al. [24] define the parameters $\alpha, c_{0}$ and $c_{1}$ as the linear function of the efficient voltage, $u$, in order to predict the behaviour of the MR damper for a fluctuating magnetic field:

$\alpha=\alpha_{a}+\alpha_{b} u$

$c_{0}=c_{0 a}+c_{0 b} u$

$c_{1}=c_{1 a}+c_{1 b} u$

To accommodate the dynamics involved in the MR fluid reaching rheological equilibrium, the following first order filter is employed to calculate efficient voltage:

$\dot{u}=-\eta(u-v)$

where $v$ is the applied voltage for current generation.

A total of 14 parameters need to be adjusted in order to fit the model response to experimental data. 


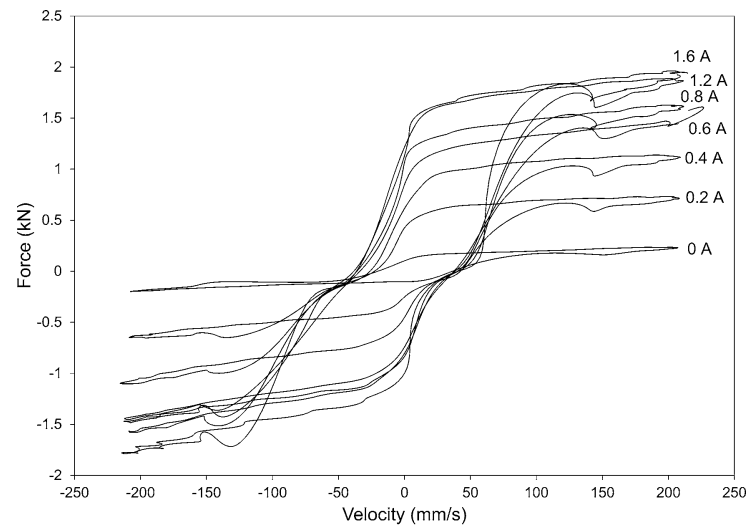

(a)

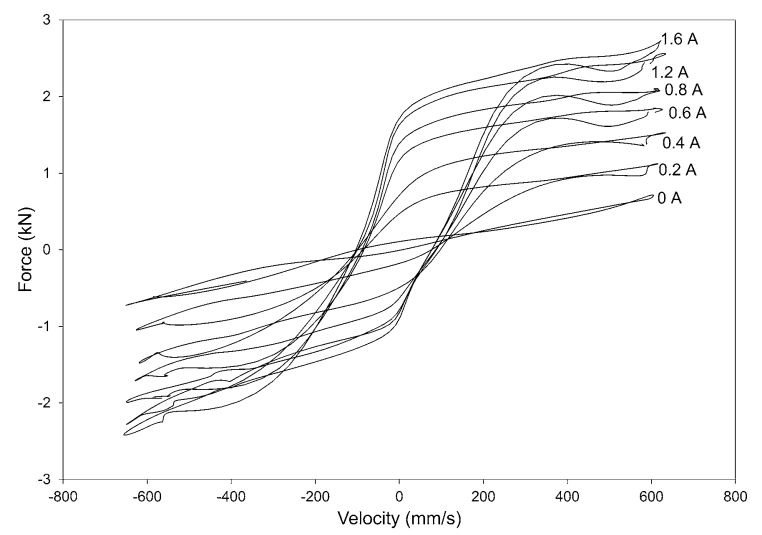

(b)

Fig. 4 Experimental MR damper curves for different levels of applied current at: a frequency $=2 \mathrm{~Hz}$ and b frequency $=6 \mathrm{~Hz}$

One of the main problems in the Bouc-Wen model is the evaluation of its characteristic parameters. To solve this problem different optimization techniques such as sequential quadratic programming algorithm [7], genetic algorithms [12, 22, 29] or charged system search optimization algorithm [26] are used to estimate the parameters that characterize the hysteresis behaviour of the MR damper. However, due to infinite solution space, these techniques demand high computational costs in order to generate the required parameters, which still cannot characterize the hysteresis behaviour of the MR damper accurately. Moreover, the state $z$ is not accessible to measurements and the only available data are the input $x$ and the output $f$ [10]. Therefore, this model is only used in applications where an accurate model is required. The Bouc-Wen model can usually be integrated using multistep or Runge-Kutta methods with adaptive stepsize [5]. In this case, there could be convergence problems with noisy inputs, i.e., when experimental data are used to fit the model response. In order to overcome this problem, different algebraic models have been proposed, such as the Kwok model. Kwok et al. [13] proposed a model that makes use of a hyperbolic tangent function to represent the hysteresis, and linear functions to represent the viscous stiffness:

$f=c \dot{x}+k x+\alpha z+f_{0}$

$z=\tanh (\beta \dot{x}+\delta \operatorname{sign}(x))$

where $c$ and $k$ are the viscous and stiffness coefficients, $\alpha$ is the scale factor of the hysteresis, $z$ is the hysteretic variable given by the hyperbolic tangent function and $f_{0}$ is the damper force offset.

The model contains only a simple hyperbolic tangent function and is computationally efficient in the context of parameter identification. Results have shown that the Kwok model was able to model responses of the MR damper and the errors were generally less than those from the Bouc-Wen model [13]. In both models, the parameters are identified for each combination of frequency of excitation and applied current. Afterwards, these parameters are fitted by polynomial functions as the only functions of the applied current. Nevertheless, the damping force will increase when the frequency of excitation increases. This means that the MR damper can respond to vibrations with different frequencies at almost the same force. As the parameters of the previous models are only functions of the applied current, the effect of the frequency is untraceable. In this paper, we propose a novel MR damper model in which parameters depend not only on the applied current, but also on the frequency of excitation.

\section{Proposed MR damper model}

Although the Kwok model has been shown to be adequate to estimate the response of an MR damper, it is necessary to continue research in order to propose new MR damper models, which are able to provide improved representation of the MR damper response. Development of an accurate mathematical model of the MR damper plays a key role in the implementation of an appropriate control strategy. In this work, a novel model based on algebraic equations is proposed to 
estimate the non-linear and hysteretic response of a MR damper. The proposed model is intended to have the following characteristics:

- The model has to improve the representation of the MR damper response in comparison with previously developed MR damper models.

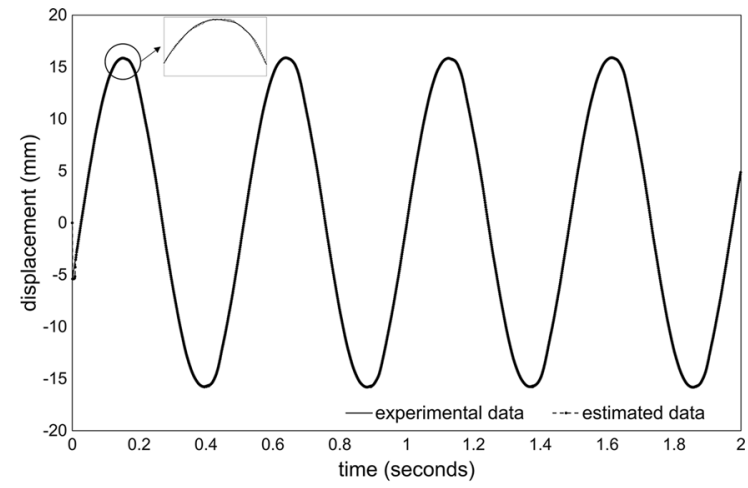

(a)

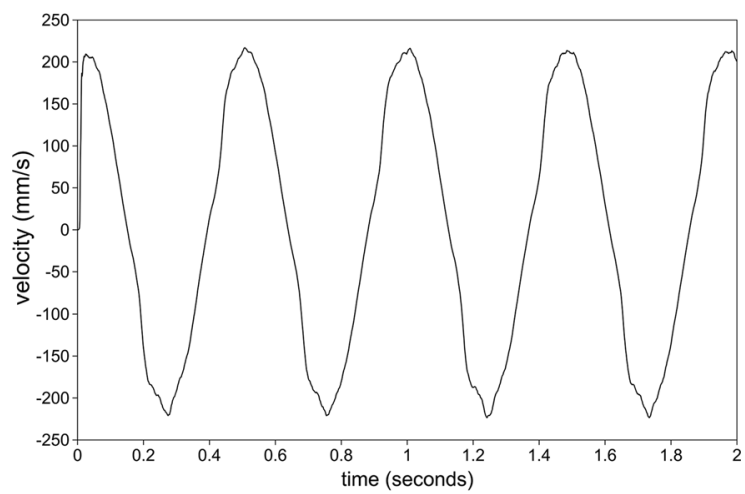

(b)

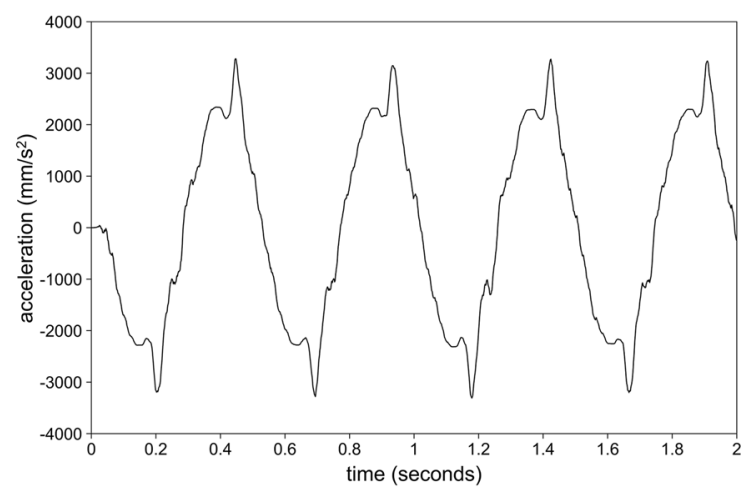

(c)

Fig. 5 a Experimental and estimated position data, b estimated velocity and $\mathbf{c}$ estimated acceleration for an input current of $0.6 \mathrm{~A}$ and frequency of $2 \mathrm{~Hz}$ using Kalman filter
- The model has to balance the best fit with the number of parameters.

- The model has to be based on algebraic equations in order not to present convergence problems even using noisy inputs. This could be a problem if experimental data are used to fit the parameters.

- The model has to take into account the variation of the parameters as a function of not only the applied current, but also the frequency of excitation.

In the literature, models have been proposed based on trigonometric functions through which responses of mechanical elements have been described [1]. One of the best known is Pacejka's Magic Formula [19] which predicts the non-linear behaviour of a tyre with a great precision. Pacejka's Magic Formula is based on 6 fitting parameters. Nevertheless, this model is not able to model hysteretic behaviour. For this reason, it is necessary to modify Pacejka's Magic Formula to add the term $k \sin g(\ddot{x})$. The term $c \dot{x}$ is also added in order to define the slope of the curve at the two ends of the hysteresis. Experimental results show that the curvature factor parameter from the original Pacejka's Magic Formula is approximately a constant with a value of 0.1 in the proposed MR damper model. Taking into account these considerations, the proposed MR damper model, based on a modified version of Pacejka's model, is formed by 7 parameters and is given by the following equations:

$f=\operatorname{Dsin}\left(\operatorname{Ctan}^{-1}(\varphi-k \operatorname{sign}(\ddot{x}))\right)+c \dot{x}+S_{v}$

$\varphi=B_{v e l}+0.1\left[\tan ^{-1}\left(B_{v e l}\right)\right]$

$B_{v e l}=B\left(\dot{x}+S_{h}\right)$

where $D, C, B, k, c, S_{h}$ and $S_{v}$ are the parameters of the model $\ddot{x}, \dot{x}$ and $x$ are the piston acceleration, velocity and displacement, respectively; $k$ is the magnitude of the hysteresis; $S_{h}$ and $S_{v}$ are the horizontal and vertical

Table 1 Lower and upper bounds for a current of $0 \mathrm{~A}$ and frequency of $2 \mathrm{~Hz}$

\begin{tabular}{llllrlll}
\hline Parameter & $D$ & $C$ & $B$ & $S_{h}$ & $S_{v}$ & $k$ & \multicolumn{1}{l}{$c$} \\
\hline Lower bound & 0.15 & 0.7 & 0.005 & -17 & 0 & 1.2 & 0.00025 \\
Upper bound & 0.2 & 1.0 & 0.1 & 10 & 0.5 & 0.8 & 0.00035 \\
\hline
\end{tabular}


Table 2 MS errors for proposed and Kwok models

\begin{tabular}{|c|c|c|c|c|c|c|c|c|}
\hline \multirow{3}{*}{$\begin{array}{l}\text { Current } \\
\text { (A) }\end{array}$} & \multicolumn{8}{|c|}{ Frequency of excitation $(\mathrm{Hz})$} \\
\hline & \multicolumn{2}{|l|}{2} & \multicolumn{2}{|l|}{4} & \multicolumn{2}{|l|}{6} & \multicolumn{2}{|l|}{8} \\
\hline & $\begin{array}{l}\text { Proposed } \\
\text { model }\end{array}$ & $\begin{array}{l}\text { Kwok } \\
\text { model }\end{array}$ & $\begin{array}{l}\text { Proposed } \\
\text { model }\end{array}$ & $\begin{array}{l}\text { Kwok } \\
\text { model }\end{array}$ & $\begin{array}{l}\text { Proposed } \\
\text { model }\end{array}$ & $\begin{array}{l}\text { Kwok } \\
\text { model }\end{array}$ & $\begin{array}{l}\text { Proposed } \\
\text { model }\end{array}$ & $\begin{array}{l}\text { Kwok } \\
\text { model }\end{array}$ \\
\hline 0 & $2.6610^{-4}$ & $3.0410^{-4}$ & $7.5610^{-4}$ & $5.6910^{-4}$ & 0.0021 & 0.0017 & 0.0063 & 0.0062 \\
\hline 0.2 & 0.0022 & 0.0027 & 0.0009 & 0.0011 & 0.0062 & 0.0066 & 0.0087 & 0.0085 \\
\hline 0.4 & 0.0048 & 0.0053 & 0.0037 & 0.0044 & 0.0052 & 0.0064 & 0.0121 & 0.0122 \\
\hline 0.6 & 0.0032 & 0.0051 & 0.0034 & 0.0055 & 0.0095 & 0.0110 & 0.0130 & 0.0137 \\
\hline 0.8 & 0.0061 & 0.0085 & 0.0103 & 0.0131 & 0.0118 & 0.0123 & 0.0146 & 0.0209 \\
\hline 1.2 & 0.0077 & 0.0104 & 0.0074 & 0.0091 & 0.0156 & 0.0176 & - & - \\
\hline 1.6 & 0.0146 & 0.0185 & 0.0184 & 0.0233 & 0.0147 & 0.0167 & - & - \\
\hline
\end{tabular}

offset values, respectively; $C$ is the shape factor which determines the shape of the peak; $D$ is the peak factor; and $B C D+c$ represents the slope of the force at low velocities. The influence of the parameters of the model is depicted in Fig. 1.

The model parameters $(D, C, B, S h, S v, k$ and $c)$ are identified for each combination of applied current and frequency of excitation using genetic algorithms (parameter identification). Then, these parameters are grouped and fitted by neural networks as functions of applied current and frequency of excitation ( $p a-$ rameter generalization). In real applications, the frequency of excitation can be calculated by measuring the velocity and the displacement of the MR damper piston [11]. Figure 2 shows the scheme of modelling for an MR damper.

\section{Experimental setup}

In order to validate the model developed in the previous section, an MR damper $R D-1005-3$ commercialized by Lord Corporation [15] is used. Experimental data are obtained from our laboratory with a damper test machine which is shown in Fig. 3. In the test machine, a hydraulic actuator is employed to drive the MR damper from sinusoidal displacement cycles with an amplitude of $16 \mathrm{~mm}$ in the $2-8 \mathrm{~Hz}$ frequency range. In the $2-6 \mathrm{~Hz}$ frequency range, the applied current was from 0 to $1.6 \mathrm{~A}$ with increment of $0.2 \mathrm{~A}$. For a frequency of excitation of $8 \mathrm{~Hz}$ and applied currents above $0.8 \mathrm{~A}$, the experimental data were not accurate enough, and did not give the desired plots. Therefore, the experiments were restricted to a maximum applied current of $0.8 \mathrm{~A}$ for this frequency of excitation.

The damper stroke was positioned at its centre before the test was carried out in order to avoid the extreme positions of the damper stroke. The damper test machine is equipped with a displacement sensor to measure the displacement $x$ of the MR damper piston and a load cell to measure the output force $f$. The signals $x$ and $f$ are sampled at the rate $1 \mathrm{kHz}$. Figure 4 shows a series of typical response curves for the MR damper for the tests corresponding to $2-6 \mathrm{~Hz}$, respectively. As observed, in the range of small velocities the force variation displays significant hysteretic behaviour, while for large velocities the force varies almost linearly with the velocity. These two distinct rheological regions over which dampers operate are known as the pre-yield and the post-yield regions [9]. As the current increases, the force required to yield the fluid increases and produces behaviour associated with a plastic material in parallel with a viscous damper. Before the maximum damper velocity is reached, the force overshooting phenomenon is observed. This phenomenon is believed to be caused by fluid inertia after the fluids yields and the fluid begins to flow [30]. Note that the non-zero mean force produced by the MR damper is due to the accumulator.

Experimental results show the variability of the MR damper response, not only with the input current, but also with the frequency of excitation. In order to estimate the force exerted by the damper from the 


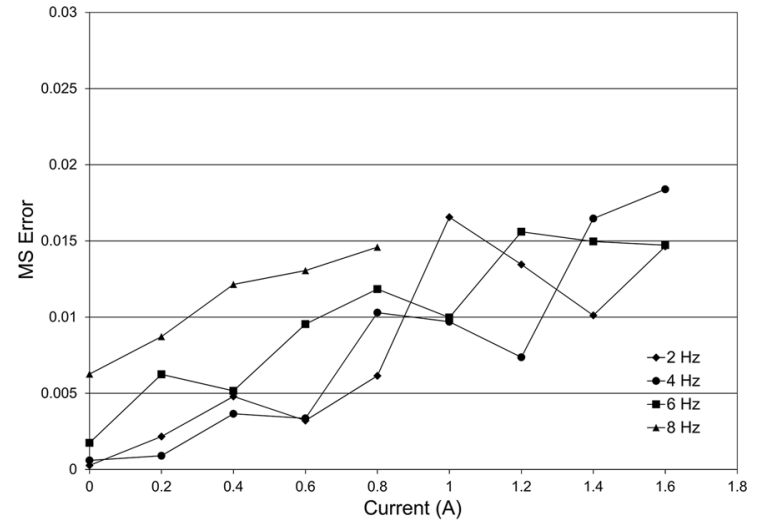

(a)

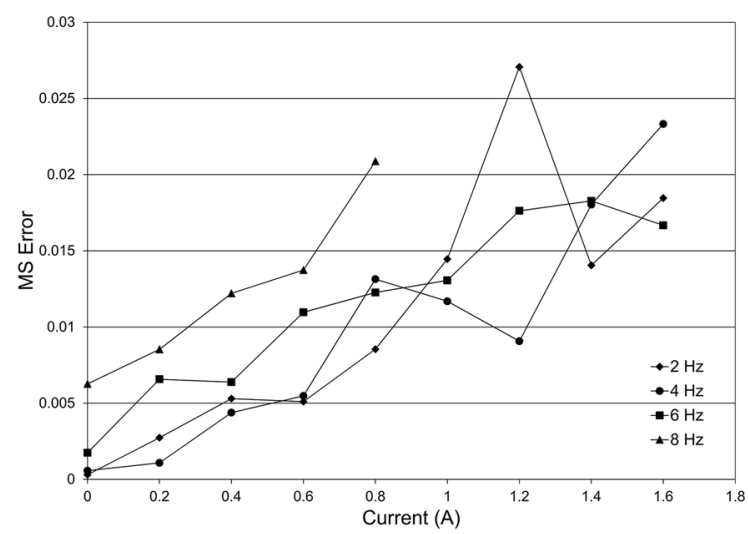

(b)

Fig. 6 Parameter identification errors a from proposed model, b from Kwok model

model, it is necessary to know not only the applied current, but also the velocity and acceleration of the MR damper piston. Although there are some prototypes for an integrated relative velocity sensor [18], at present, the best option it is to estimate both variables.

\subsection{Damper velocity and acceleration estimation from sampled position data by Kalman filter}

The simplest solution to estimate the velocity and acceleration of the MR damper piston from sampled position data is to use the finite difference (FD) method. The velocities and accelerations are calculated through the following expressions [2]:

$$
\begin{aligned}
& \dot{x}_{k}=\frac{x_{k}-x_{k-1}}{T} \\
& \ddot{x}_{k}=\frac{x_{k}-2 x_{k-1}+x_{k-2}}{T^{2}}
\end{aligned}
$$

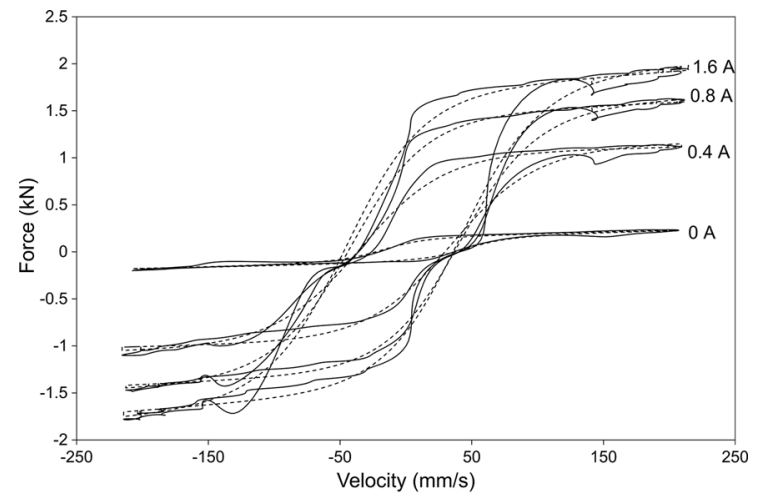

(a)

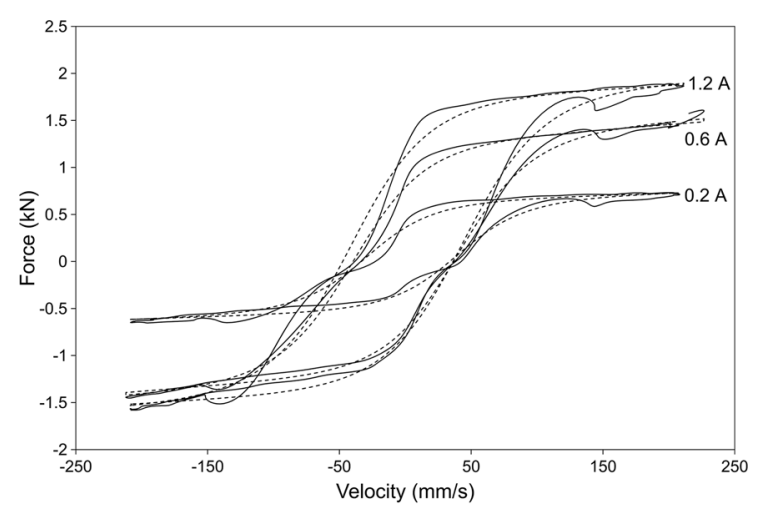

(b)

Fig. 7 Comparison between the experimental (solid lines) and estimated (dashed lines) responses with fitted parameters from proposed model and with applied current ranged from 0 to $1.6 \mathrm{~A}$ and frequency of $2 \mathrm{~Hz}$

Then, the results are filtered using a low-pass filter. The main problem is that this method does not achieve suitable results. The Kalman filter has been shown to be an adequate candidate to estimate the velocity and acceleration from position data [20]. This filter is a mathematical tool that is used for stochastic estimation

from noisy sensor measurements. The nonlinear system governed by the nonlinear stochastic difference equations can be written as:

$\mathbf{x}_{k+1}=f_{k}\left(\mathbf{x}_{k}, \mathbf{u}\right)+\mathbf{w}_{k}$

$\mathbf{y}_{k}=h_{k}\left(\mathbf{x}_{k}, \mathbf{u}\right)+\mathbf{v}_{k}$

where $\mathbf{x}_{k}$ is the state vector, $\mathbf{u}$ is the input vector, $\mathbf{w}_{k}$ is the process noise vector, $\mathbf{y}_{k}$ is the measurement vector and $\mathbf{v}_{k}$ is the measurement noise vector. $\mathbf{w}_{k}$ and $\mathbf{v}_{k}$ are assumed to be white, zero mean and uncorrelated: 


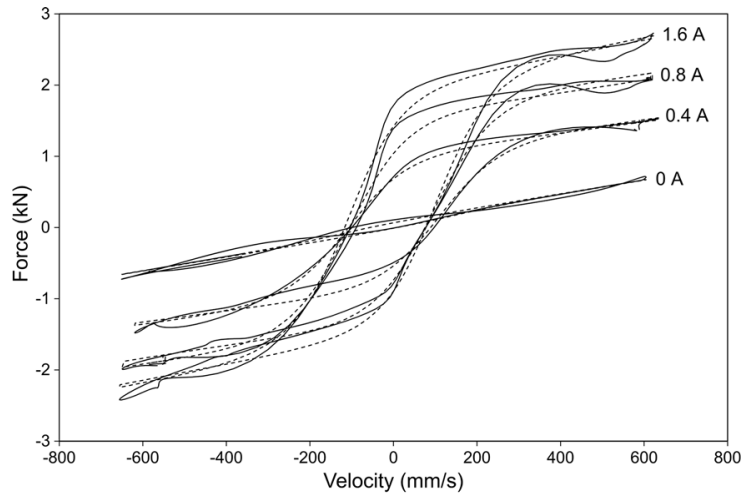

(a)

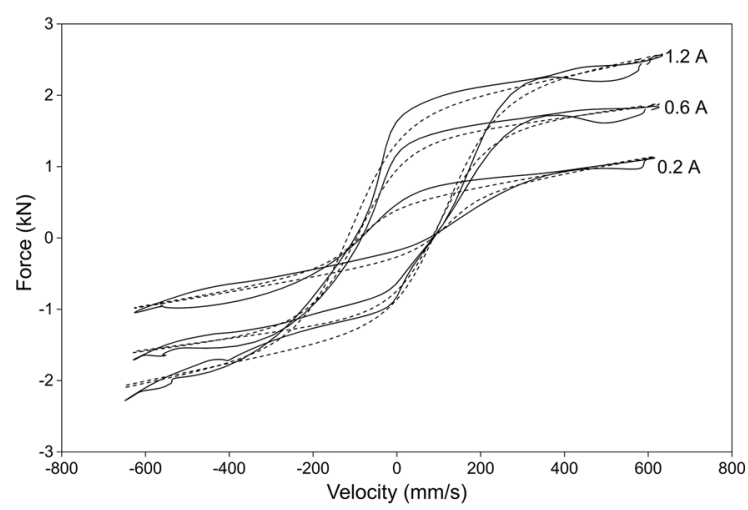

(b)

Fig. 8 Comparison between the experimental (solid lines) and estimated (dashed lines) responses with fitted parameters from proposed model and with applied current ranged from 0 to $1.6 \mathrm{~A}$ and frequency of $6 \mathrm{~Hz}$

$\mathbf{w}_{k} \sim N\left(0, \mathbf{Q}_{k}\right)$

$\mathbf{v}_{k} \sim N\left(0, \mathbf{R}_{k}\right)$

where $\mathbf{Q}$ and $\mathbf{R}$ are the covariance matrices describing the second-order properties of the state and measurement noise. For the particular case of velocity and acceleration estimation from displacement data, the discrete-time system can be written as [20]:

$$
\begin{aligned}
& {\left[\begin{array}{c}
x_{k+1} \\
\dot{x}_{k+1} \\
\ddot{x}_{k+1}
\end{array}\right]=\left[\begin{array}{ccc}
1 & T & T^{2} / 2 \\
0 & 1 & T \\
0 & 0 & 1
\end{array}\right]\left[\begin{array}{c}
x_{k} \\
\dot{x}_{k} \\
\ddot{x}_{k}
\end{array}\right]+\left[\begin{array}{c}
T^{3} / 6 \\
T^{2} / 2 \\
T
\end{array}\right]} \\
& y_{k}=x_{k}+v_{k}
\end{aligned}
$$

where $T$ is the sample time period and the covariance matrices are:

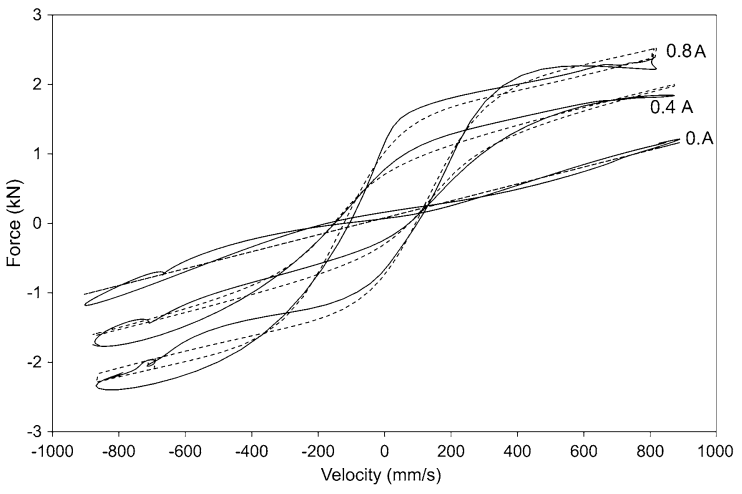

(a)

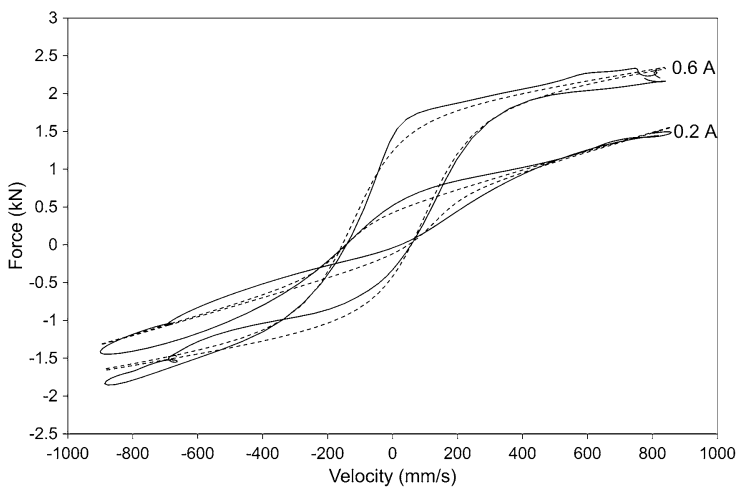

(b)

Fig. 9 Comparison between the experimental (solid lines) and estimated (dashed lines) responses with fitted parameters from proposed model and with applied current ranged from 0 to $0.8 \mathrm{~A}$ and frequency of $8 \mathrm{~Hz}$

$\mathbf{Q}=200\left[\begin{array}{ccc}T^{5} / 20 & T^{4} / 8 & T^{3} / 6 \\ T^{4} / 8 & T^{3} / 6 & T^{2} / 2 \\ T^{3} / 6 & T^{2} / 2 & T\end{array}\right]$

$R=\frac{0.1^{2}}{1+\dot{x}_{k}}$

The Kalman filter is summarized as the following recursive equations:

1. The prediction of the state given by:

$$
\tilde{\mathbf{x}}_{k \mid k-1}=\mathbf{A} \mathbf{x}_{k-1 \mid k-1}+\mathbf{B} \mathbf{u}_{k}
$$

2. The predicted error covariance is computed as:

$$
\mathbf{P}_{k \mid k-1}=\mathbf{A} \mathbf{P}_{k-1 \mid k-1} \mathbf{A}^{T}+\mathbf{Q}
$$

3. The Kalman gain is calculated by:

$$
\mathbf{K}_{x}=\mathbf{P}_{k \mid k-1}+\mathbf{H}^{T}\left(\mathbf{H} \mathbf{P}_{k \mid k-1} \mathbf{H}^{T}+\mathbf{R}\right)^{-1}
$$

4. The state estimation is updated with measurement $\mathbf{y}_{k}$ : 


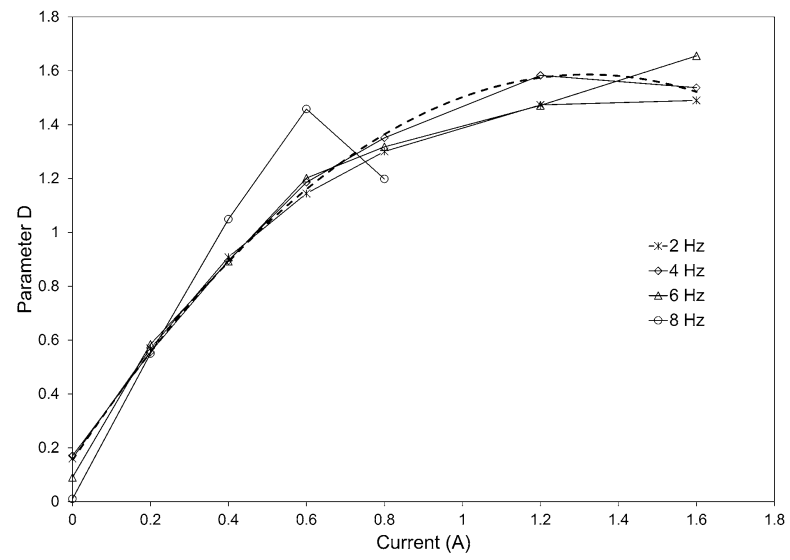

(a)

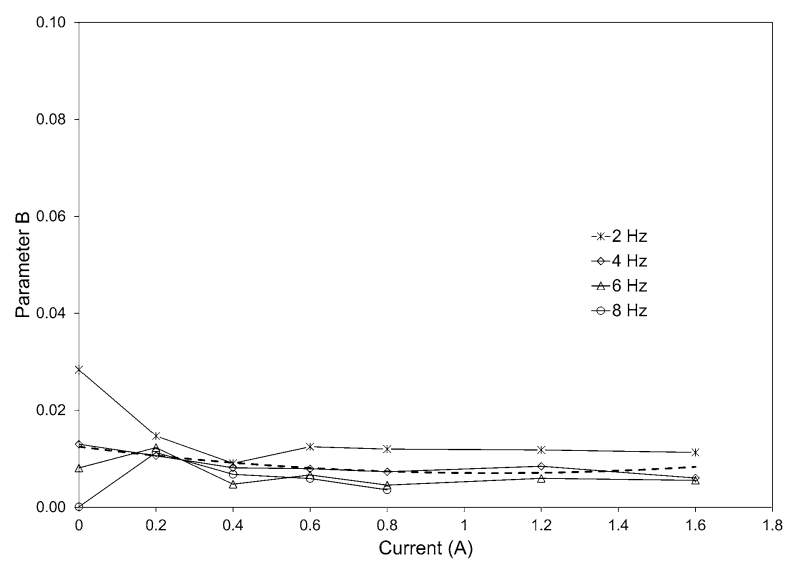

(c)

Fig. 10 Parameter identification results (solid lines) and polynomial fitted (dashed line) from proposed model versus the applied current for different frequencies. a Parameter $D$,

$$
\tilde{\mathbf{x}}_{k \mid k}=\tilde{\mathbf{x}}_{k \mid k-1}+\mathbf{K}_{x}\left(\mathbf{y}_{k}-\mathbf{H} \mathbf{x}_{k \mid k-1}\right)
$$

5. Finally, the error covariance is updated:

$$
\mathbf{P}_{k \mid k}=\left(\mathbf{I}-\mathbf{K}_{x} \mathbf{H}\right) \mathbf{P}_{k \mid k-1}
$$

In Fig. 5 the experimental and estimated position data (a), estimated velocity (b) and estimated acceleration (c) for an input current of $0.6 \mathrm{~A}$ and frequency of $2 \mathrm{~Hz}$ using the Kalman filter are shown.

\section{Results and discussion}

The results obtained for parameter identification and parameter generalization steps, as is shown in Fig. 2, are presented in this section.

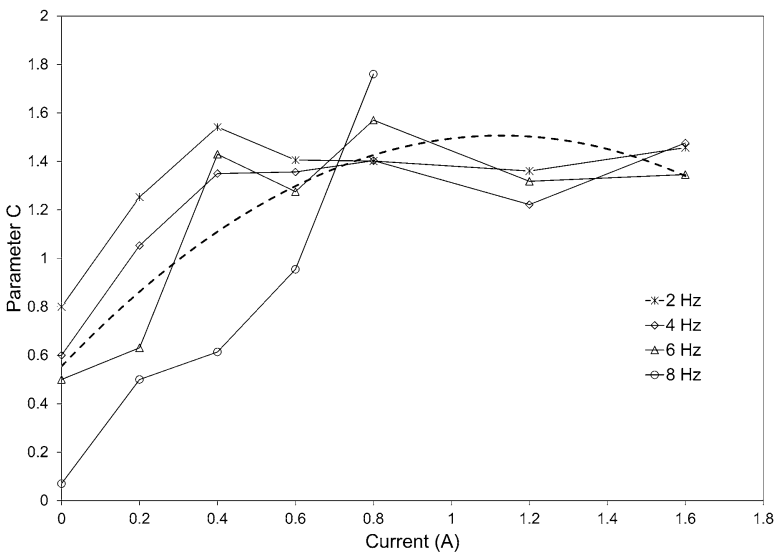

(b)

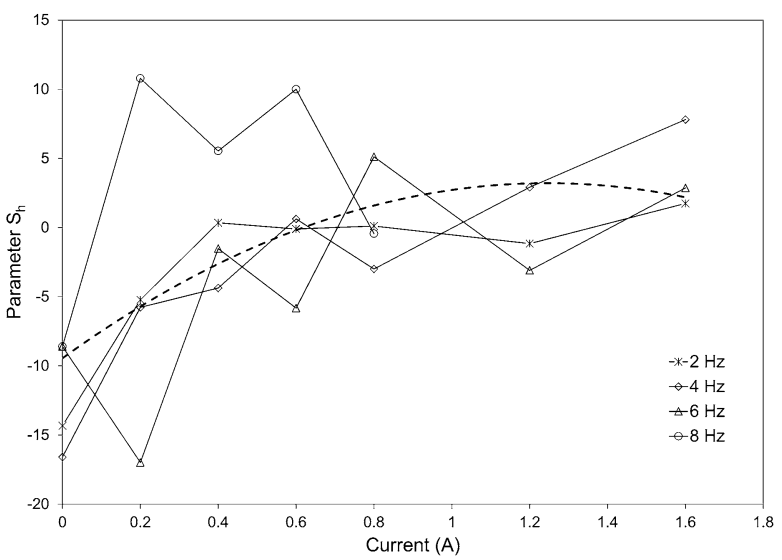

(d)

b Parameter $C$, c Parameter $B$, d Parameter $S_{h}$, e Parameter $S_{v}$, f Parameter $k, \mathbf{g}$ Parameter $c$

\subsection{Parameter identification}

Genetic algorithms (GAs) are a heuristic search and optimization technique inspired by natural evolution. The genetic algorithm repeatedly modifies a population of individual solutions called chromosomes. At each step, the genetic algorithm selects individuals from the current population to be parents and uses them to produce children for the next generation by means of the evaluation of an objective function (fitness function). The cycle of evolution is repeated until a desired termination criterion is reached. This criterion can be set by the number of evolution cycles, or the amount of variation of individuals between different generations, or a predefined value of fitness. The best chromosome generated during the search is 


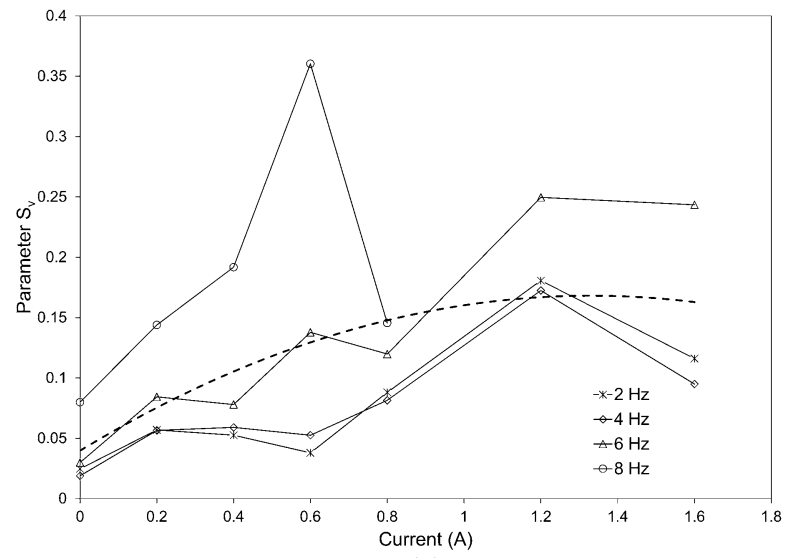

(e)

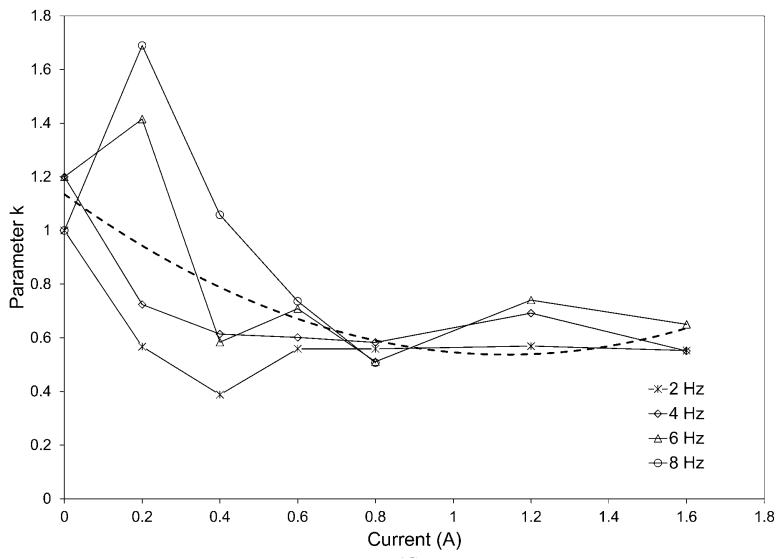

(f)

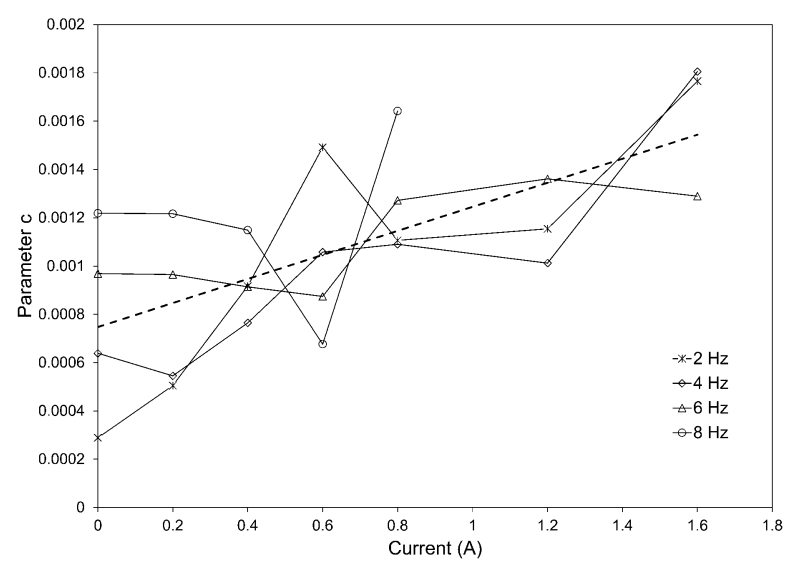

(g)

Fig. 10 continued

the final result of the genetic algorithm. GAs have been successfully applied to a wide range of realworld problems of significant complexity. Their advantages are that:

- They are particularly well-suited to multidimensional global search problems where the search space potentially contains the multiple local minimum;

- a basic GA does not require extensive knowledge of the search space, reducing computing time; and

- GAs seek good solutions and combine them in order to obtain better solutions.

In this work, Genetic Algorithm Toolbox for Matlab was used. The parameters to be identified are $D, C, B$, $S_{h}, S_{v}, k$ and $c$, hence, the chromosome becomes
$\mathbf{C}_{i}=\left\{D_{i}, C_{i}, B_{i}, S_{h i}, S_{v i}, k_{i}, c_{i}\right\} \quad i=1, \ldots, N$

where $N$ is the population size. The population type, which specifies the type of the input to the fitness function, is chosen as a double vector. After analysing the influence of the model parameters on the shape of the damper force-velocity curve (Fig. 1), the lower and upper bounds of all parameters are defined from the experimental MR damper curves. As an example, Table 1 represents the lower and upper bounds for a current of $0 \mathrm{~A}$ and frequency of $2 \mathrm{~Hz}$. In order to obtain the optimal values for the parameters of the proposed model, the fitness function is evaluated as the mean-square error between the experimental and estimated damper forces: 


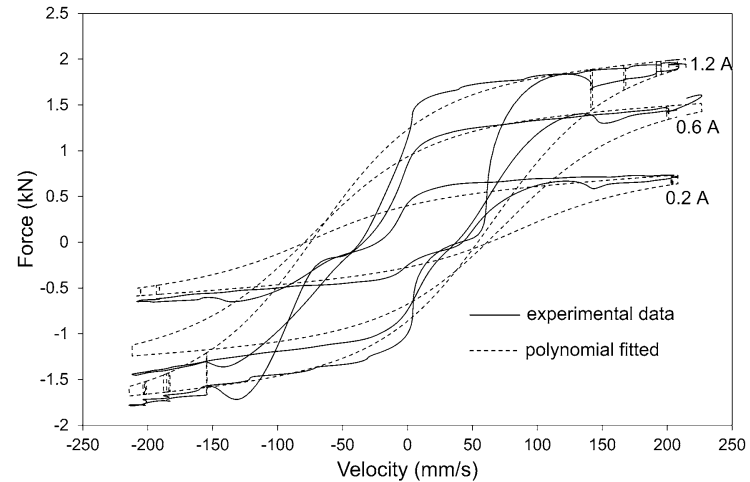

(a)

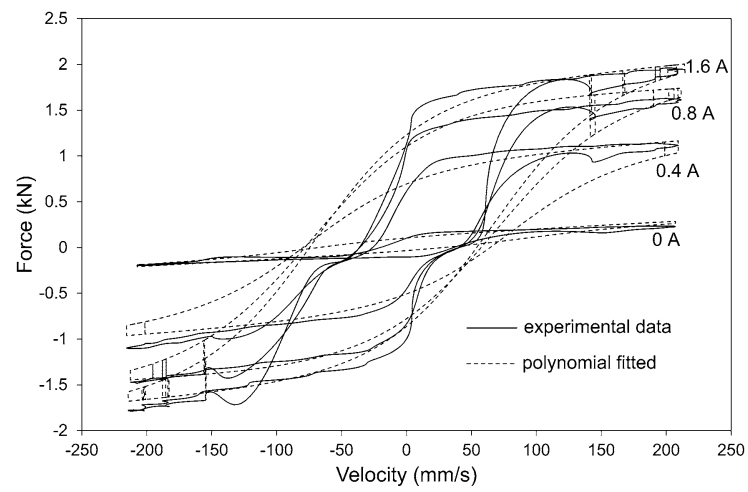

(b)

Fig. 11 Comparison between the experimental and estimated responses from proposed model with polynomial fitted parameters (frequency independent model) for an applied current ranged from 0 to $1.6 \mathrm{~A}$ and frequency of $2 \mathrm{~Hz}$

$f_{\text {fitness }}=\frac{1}{N} \sum_{i=1}^{N}\left(F_{\exp }^{i}-F_{\text {est }}^{i}\right)^{2}$

where $F_{\text {exp }}$ is the measured or experimental force, $F_{\text {est }}$ is the force estimated by the proposed model, and $N$ is the number of experimental measurements.

Nonlinear constraints are also defined to limit the feasible search region:

$m(1-15 \%)<B C D+c<m(1+15 \%)$

where $m$ is the slope at low velocities.

Mean-Squared Error results of the proposed model are given in Table 2 and Fig. 6. In order to validate the proposed model, errors are compared with the errors obtained from the Kwok model [13]. It can be observed that errors from the proposed model have smaller values than the Kwok model. Figures 7, 8 and 9 compare the force-velocity obtained by the

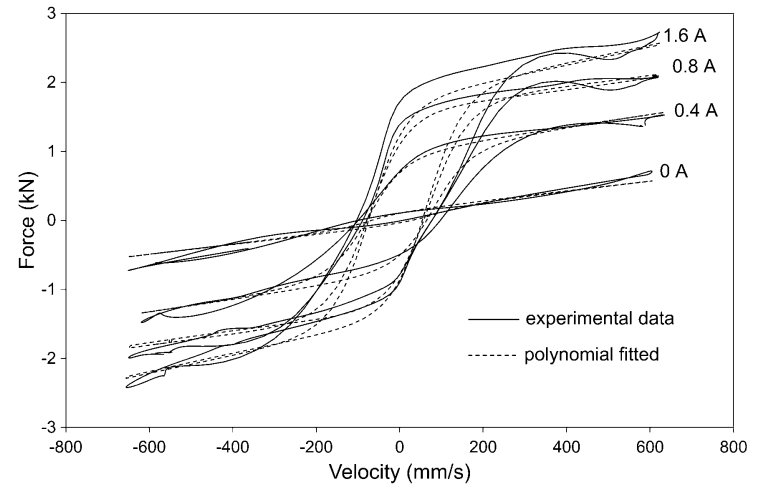

(a)

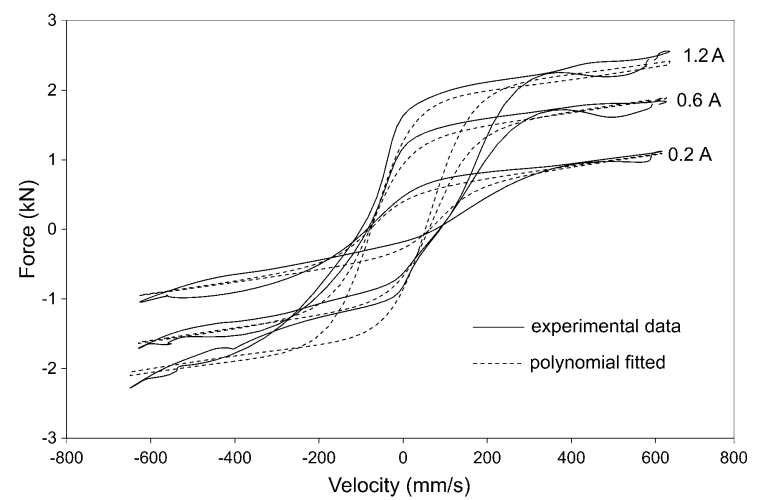

(b)

Fig. 12 Comparison between the experimental and estimated responses from proposed model with polynomial fitted parameters (frequency independent model) for an applied current ranged from 0 to $1.6 \mathrm{~A}$ and frequency of $6 \mathrm{~Hz}$

experimental test for the frequencies of 2,6 and $8 \mathrm{~Hz}$ with those modelled by the proposed model. The examination of these figures reveals that there is excellent agreement between the simulation and the experimental results.

\subsection{Parameter generalization}

A literature survey indicates that in most of the previously developed models, the variation of the identified parameters for different supplied currents is modelled by polynomial functions [12, 13, 23]. According to this, relationships between the averaged values of the identified parameters of the proposed model and the supplied current are given by the following expressions (Fig. 10):

$D=-0.8226 i^{2}+2.1713 i+0.1532$ 
$C=-0.7485 i^{2}+1.6884 i+0.5544$

$B=0.0047 i^{2}-0.0101 i+0.0125$

$S_{h}=-8.1556 i^{2}+20.321 i-9.452$

$S_{v}=-0.0726 i^{2}+0.1931 i+0.0397$

$k=0.4617 i^{2}-1.0508 i+1.1351$

$c=0.0005 i+0.0007$

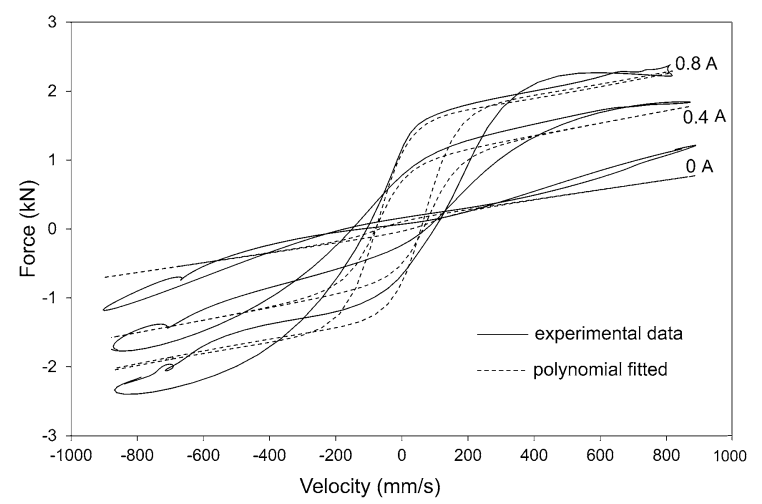

(a)

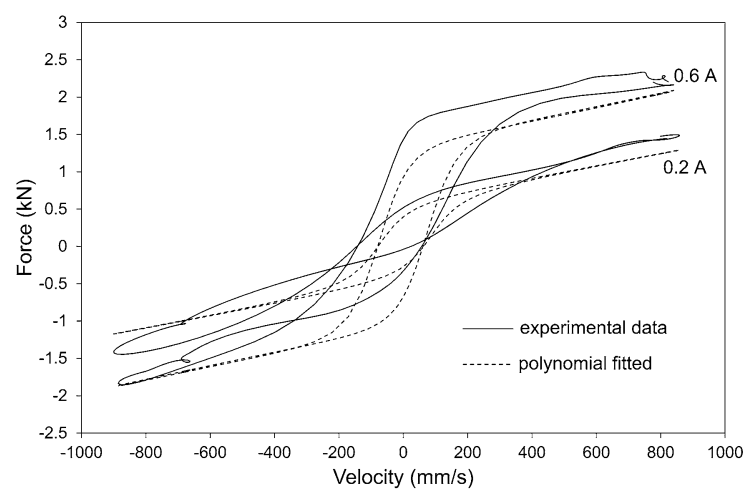

(b)

Fig. 13 Comparison between the experimental and estimated responses from proposed model with polynomial fitted parameters (frequency independent model) for an applied current ranged from 0 to $0.8 \mathrm{~A}$ and frequency of $8 \mathrm{~Hz}$
Figures 11, 12 and 13 show the results from the polynomial fitting as functions of the supplied current. Examination of these figures reveals significant disagreement between the results of the proposed frequency independent model (polynomial fitting) and the experimental ones. The results show a significant dependency, not only with the current but also with the frequency. Dominguez et al. [6] proposed a modified Bouc-Wen model where the frequency of excitation and applied current have been incorporated as variables to the model. Although an excellent agreement between the simulated results from the model and the

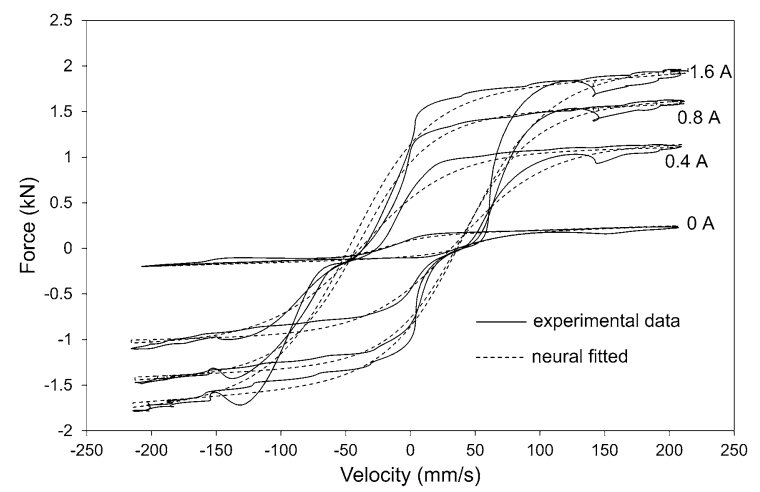

(a)

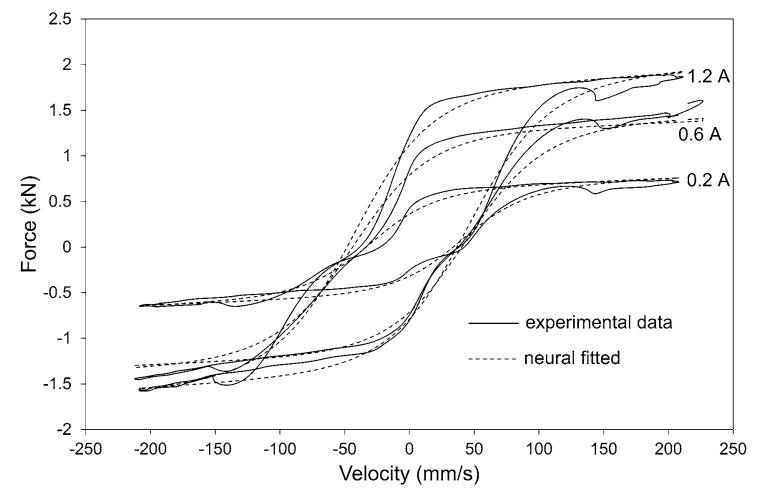

(b)

Fig. 14 Comparison between the experimental and estimated responses from proposed model with neural fitted parameters (frequency dependent model) for an applied current ranged from 0 to $1.6 \mathrm{~A}$ and frequency of $2 \mathrm{~Hz}$

Table 3 Mean squared error of the neural networks train

\begin{tabular}{llllllll}
\hline Parameter & $D$ & $C$ & $B$ & $S_{h}$ & $S_{v}$ & $k$ & $c$ \\
\hline MSE & $2.2610^{-23}$ & $3.3810^{-21}$ & $1.1210^{-7}$ & $3.3410^{-20}$ & $7.6710^{-20}$ & $1.9610^{-20}$ & $3.8110^{-8}$ \\
\hline
\end{tabular}




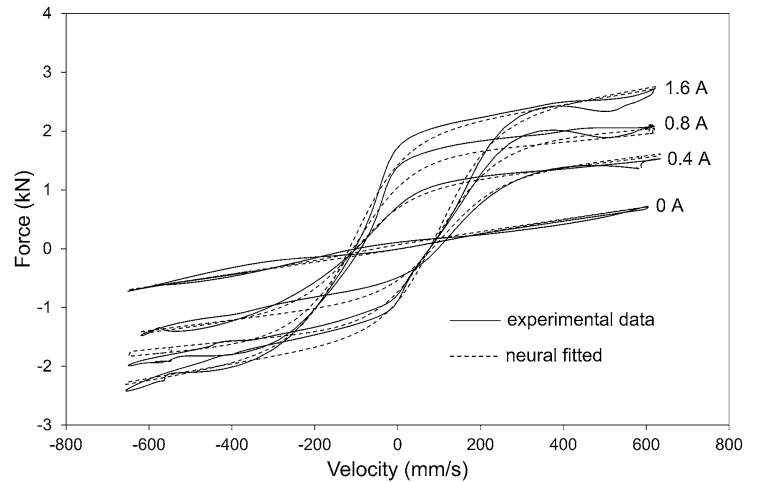

(a)

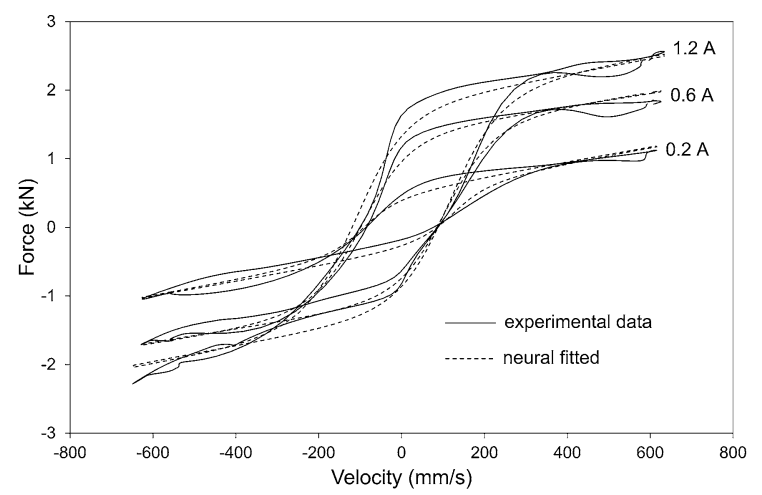

(b)

Fig. 15 Comparison between the experimental and estimated responses from proposed model with neural fitted parameters (frequency dependent model) for an applied current ranged from 0 to $1.6 \mathrm{~A}$ and frequency of $6 \mathrm{~Hz}$

experimental results was demonstrated, the number of parameters to fit is high (20 parameters); this may cause difficulties in their identification. To solve this problem, in this work, the use of neural networks to model the parameters identified from the optimization algorithm as functions of applied current and frequency of excitation is proposed.

Neural networks (NN) have been effectively applied to model complex systems due to their good learning capability. The goal of neural network training is to iteratively update the network weights to minimize the learning error. Among NN architectures, a class of Multi-Layer Perceptron (MLP) has become the most popular architecture, due to its ability to model simple, as well as complex, functional relationships. The MLP is composed of layers of nodes: input layer, output layer and a number of hidden layers. Typically, one hidden layer is sufficient

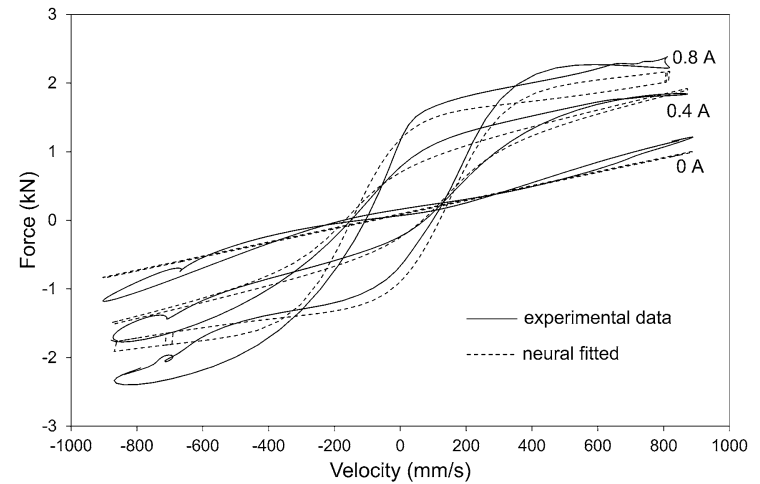

(a)

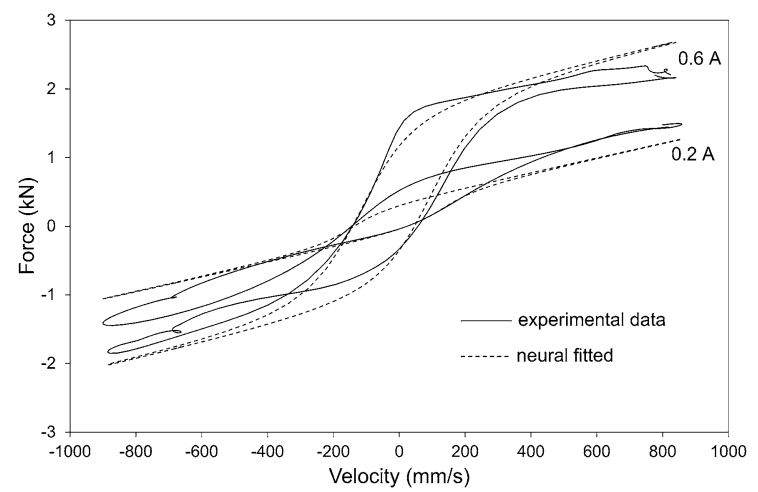

(b)

Fig. 16 Comparison between the experimental and estimated responses from proposed model with neural fitted parameters (frequency dependent model) for an applied current ranged from 0 to $0.8 \mathrm{~A}$ and frequency of $8 \mathrm{~Hz}$

for most practical applications. As for the number of nodes in each layer, the number of input layer nodes is equal to the dimension of the input vector, the number of output nodes is usually equal to the dimension of the output vector and the number of hidden nodes is usually decided using trial and error. The estimated output, $\tilde{y}_{i}$, can be expressed as

$\left.\tilde{y}_{i}=F_{i}\left[\sum_{j=1}^{q} W_{i j} f_{i} \sum_{k=1}^{p} w_{j k} u_{k}+w_{j 0}\right)+W_{i 0}\right]$

where $F_{i}$ and $f_{i}(i=1,2, \ldots, m)$ are the activation functions of the output and hidden layers, respectively; $W_{i j}$ and $w_{j k}(j=1,2, \cdots, q ; k=1,2, \cdots, p)$ are the weights of the output and hidden layers, respectively; and $W_{i 0}$ and $w_{j 0}$ are the bias of the output and hidden layers, respectively; $p$ is the size of the input vector $\mathbf{u} ; q$ is the size of the hidden nodes; and $m$ is the dimension of the output vector. The hyperbolic tangent sigmoid is 
Table 4 MS Errors for proposed model from polynomial fitted parameters (frequency independent model) and neural fitted parameters (frequency dependent model)

\begin{tabular}{|c|c|c|c|c|c|c|c|c|}
\hline \multirow{3}{*}{$\begin{array}{l}\text { Current } \\
\text { (A) }\end{array}$} & \multicolumn{8}{|c|}{ Frequency of excitation $(\mathrm{Hz})$} \\
\hline & \multicolumn{2}{|l|}{2} & \multicolumn{2}{|l|}{4} & \multicolumn{2}{|l|}{6} & \multicolumn{2}{|l|}{8} \\
\hline & $\mathrm{NN}$ & Polynomial & $\mathrm{NN}$ & Polynomial & $\mathrm{NN}$ & Polynomial & $\mathrm{NN}$ & Polynomial \\
\hline 0 & 0.0005 & 0.0014 & 0.0042 & 0.0011 & 0.0026 & 0.0097 & 0.0109 & 0.0613 \\
\hline 0.2 & 0.0025 & 0.0245 & 0.0015 & 0.0034 & 0.0071 & 0.0074 & 0.0129 & 0.034 \\
\hline 0.4 & 0.0048 & 0.0517 & 0.0038 & 0.0083 & 0.0078 & 0.0129 & 0.0123 & 0.0426 \\
\hline 0.6 & 0.0098 & 0.0841 & 0.0045 & 0.0115 & 0.0152 & 0.015 & 0.0819 & 0.0888 \\
\hline 0.8 & 0.0065 & 0.0583 & 0.0109 & 0.0166 & 0.0198 & 0.0202 & 0.1059 & 0.0567 \\
\hline 1.2 & 0.0081 & 0.0493 & 0.0088 & 0.0133 & 0.0171 & 0.0589 & - & - \\
\hline 1.6 & 0.0147 & 0.0712 & 0.0242 & 0.0242 & 0.0164 & 0.0477 & - & - \\
\hline
\end{tabular}

selected as activation function in the hidden layer. The set of parameters to learn is $\mathbf{w}=\{W i j, w j k, W i 0, w j 0\}$.

MLP utilizes a supervised learning technique called backpropagation for training the network that minimizes the error function. Here, the network training function trainlm of Neural Networks Toolbox for Matlab has been used. This function updates weight and bias values according to Levenberg-Marquardt optimization:

$\mathbf{w}^{k+1}=\mathbf{w}^{k}-\left[\mathbf{J}^{T} \mathbf{J}+\mu \mathbf{I}\right]^{-1} \mathbf{J}^{T} \mathbf{e}$

where $\mu$ is the learning rate, $\mathbf{J}$ is the Jacobian matrix that contains first derivatives of the network errors with respect to the weights and biases, and e is a vector of Mean Squared normalized Errors (MSE) defined as:

$\mathbf{e}=\frac{1}{N} \sum_{l=1}^{N}\left(\mathbf{y}_{l}-\tilde{\mathbf{y}}_{l}\right)^{2}$

where $N$ is the number of patterns; and $\mathbf{y}_{l}$ is the target output. Here, seven neural networks have been defined, each one corresponding to an identified parameter. The inputs are the applied current and the frequency of excitation. The size of the hidden layer is 9. The performance of the neural networks after training is depicted in Table 3.

Figures 14, 15 and 16 show neural networks are an adequate candidate for modelling the relationship between the parameters and the current and frequency. Comparison between Mean-Squared Error results from the polynomial fitting (frequency independent model) and neural fitting (frequency dependent model) is shown in Table 4.

\section{Conclusions}

This paper presents a novel frequency dependent model for an MR damper. The proposed model is based on trigonometric functions, which have been shown to be adequate candidates for predicting the behaviour of mechanical systems. Results have shown the estimated model satisfactorily emulates the hysteretic and inherent non-linear behaviour of the MR damper. There are significant advantages gained by this new method concerning previous proposed models. In comparison with the Bouc-Wen model, the most commonly-used model, only seven model parameters have to be identified, decreasing the complexity and increasing the efficiency of the identification problem. Unlike MR damper models based on differential equations, the proposed model does not have convergence problems when noisy inputs from experimental measurements are used. Neural networks are used to model the relationship between the model parameters and the applied current and frequency of excitation. Finally, experimental data are used to illustrate the effectiveness of the proposed model. Results confirm that the proposed frequency dependent MR damper model improves the accuracy of the model in force simulation. 
Acknowledgements Authors gratefully acknowledge the funds provided by the Spanish Government through the CICYT Projects TRA2008-05373/AUT and TRA2011-28548C02-01.

\section{References}

1. Balamurugan L, Jancirani J, Eltantawie MA (2014) Generalized magnetorheological (MR) damper model and its application in semi-active control of vehicle suspension system. Int J Automot Technol 15(3):419-427

2. Belanger PR (1992) Estimation of angular velocity and acceleration from shaft encoder measurements. In: Proceedings of the 1992 IEEE international conference on robotics and automation, 1992. pp 585-592

3. Boada M, Calvo J, Boada B, Diaz V (2011) Modeling of a magnetorheological damper by recursive lazy learning. Int J Non-Linear Mech 46(3):479-485

4. Choi SB, Han YM (2005) Hysteretic behavior of a magnetorheological fluid: experimental identification. Acta Mech 180(1):37-47

5. Dimian M, Andrei P (2014) Mathematical models of hysteresis. Noise-driven phenomena in hysteretic systems. Springer, Berlin, pp 1-63

6. Dominguez A, Sedaghati R, Stiharu I (2006) A new dynamic hysteresis model for magnetorheological dampers. Smart Mater Struct 15(5):1179

7. Dyke SJ, Spencer BF Jr, Sain MK, Carlson JD (1996) Modeling and control of magnetorheological dampers for seismic response reduction. Smart Mater Struct 5(5):565-575

8. Gamota DR, Filisko FE (1991) Dynamic mechanical studies of electrorheological materials: moderate frequencies. J Rheol 35:399-425

9. Giuclea M, Sireteanu T, Stancioiu D, Stammers CW (2004) Model parameter identification for vehicle vibration control with magnetorheological dampers using computational intelligence methods. Proc Inst Mech Eng, Part I J Syst Contr Eng 218(7):569-581

10. Ikhouane F, Rodellar J (2005) On the hysteretic Bouc-Wen model. Nonlinear Dyn 42(1):79-95

11. Jahromi AF, Bhat RB, Xie WF (2015) Frequency dependent spencer modeling of magnetorheological damper using hybrid optimization approach. Shock Vib 2015:8

12. Kwok NM, Ha QP, Nguyen MT, Li J, Samali B (2007) Bouc-Wen model parameter identification for a MR fluid damper using computationally efficient GA. ISA Trans 46(2):167-179

13. Kwok NM, Ha QP, Nguyen TH, Li J, Samali B (2006) A novel hysteretic model for magnetorheological fluid dampers and parameter identification using particle swarm optimization. Sens Actuators A 132(2):441-451

14. Li Z, Zheng J, Koo JH, Wang J (2013) An improved polynomial dynamic model of a magnetorheological fluid damper under impact loading. In: Proceedings of the SPIE 8688 , active and passive smart structures and integrated systems, vol. $86881 \mathrm{C}$

15. Lord Corporation (2015) http://www.lord.com/

16. Metered H, Bonello P, Oyadiji S (2015) Nonparametric identification modeling of magnetorheological damper using chebyshev polynomials fits. SAE Int J Passeng Cars Mech Syst 2(1):1125-1135

17. Min CK, Lee HJ, Cho SW, Lee IW (2007) Modified energy dissipation algorithm for seismic structures using magnetorheological damper. KSCE J Civil Eng 11(2):121-126

18. Nehl TW, Betts JA, Mihalko LS (1995) An integrated relative velocity sensor for real time damping applications. In: Industry applications conference, 1995. Thirtieth IAS annual meeting, IAS'95, vol. 1, pp 484-491

19. Pacejka H (2012) Tire and vehicle dynamics, 3rd edn. Elsevier, Amsterdam

20. Puglisi L, Saltaren R, Garcia-Cena C (2015) On the velocity and acceleration estimation from discrete time-position signal of linear encoders. J Control Eng Appl Inform 17(3):30-40

21. Segla S, Orecny M (2014) Balance control of semiactive seat suspension with elimination of dynamic jerk. Procedia Eng 96:419-427

22. Sireteanu T, Giuclea M, Mitu AM (2010) Identification of an extended Bouc-Wen model with application to seismic protection through hysteretic devices. Comput Mech 45(5):431-441

23. Spaggiari A, Dragoni E (2012) Efficient dynamic modelling and characterization of a magnetorheological damper. Meccanica 47(8):2041-2054

24. Spencer B, Dyke S, Sain M, Carlson J (1997) Phenomenological model for magnetorheological dampers. J Eng Mech 123(3):230-238

25. Strecker Z, Mazrek I, Roupec J, Klapka M (2015) Influence of MR damper response time on semiactive suspension control efficiency. Meccanica 50(8):1949-1959

26. Talatahari S, Kaveh A, Rahbari NM (2012) Parameter identification of Bouc-Wen model for MR fluid dampers using adaptive charged system search optimization. J Mech Sci Technol 26(8):2523-2534

27. Wang W, Song Y (2012) Nonlinear vibration semi-active control of automotive steering using magneto-rheological damper. Meccanica 47(8):2027-2039

28. Wereley N, Pang L, Kamath G (1998) Idealized hysteresis modeling of electrorheological and magnetorheological dampers. J Intell Mater Syst Struct 9(8):642-649

29. Xiaomin X, Qing S, Ling Z, Bin Z (2009) Parameter estimation and its sensitivity analysis of the MR damper hysteresis model using a modified genetic algorithm. J Intell Mater Syst Struct 20:2089-2100

30. Yang G, Jung HJ, Spencer BF, Carlson JD (2004) Dynamic modeling of large-scale magnetorheological damper systems for civil engineering applications. J Eng Mech 9:1107-1114 